\author{
Guang-Huar Young $\cdot$ Han-Min Chen $\cdot$ Chi-Tsai Lin \\ Kuang-Ching Tseng $\cdot$ Jiann-Shing Wu \\ Rong-Huay Juang
}

\title{
Site-specific phosphorylation of L-form starch phosphorylase by the protein kinase activity from sweet potato roots
}

Received: 5 May 2005/ Accepted: 29 June 2005/Published online: 3 September 2005

(C) Springer-Verlag 2005

\begin{abstract}
A 78-amino acid insertion (L78) is found in the low-affinity type (L-form) of starch phosphorylase (LSP, EC 2.4.1.1). This insertion blocks the starch-binding site on the L-SP molecule, and it decreases the binding affinity of L-SP toward starch. The computational analysis of the amino acid sequence on L78 predicts several phosphorylation sites at its Ser residues. Indeed, from the immunoblotting results using antibodies against phosphoamino acids, we observed that the purified L-SP from mature sweet potato (Ipomoea batatas) roots is phosphorylated. This observation led us to the detection of a protein kinase activity in the protein fraction of the crude extract from the sweet potato roots. The kinase was partially purified by liquid chromatography, and its native molecular mass was estimated as $338 \mathrm{kDa}$. An expressed peptide (L78P) containing the essential part of L78 was intensively phosphorylated by the kinase. However, H-SP (the high-affinity isomer of SP lacking the L78 insertion) and the proteolytic modified L-SP, which lost its L78 fragment, could not be phosphorylated. Furthermore, using L78P mutants by site-directed mutagenesis at Ser residues on L78, we demonstrate that only one Ser residue on L78 is phosphorylated by the kinase. These results imply that this kinase is specific to L-SP, or more precisely, to the L78 insertion. The in vitro phosphorylated L-SP shows
\end{abstract}

H.M. Chen and C.T. Lin contributed equally to this work.

G.-H. Young · K.-C. Tseng · J.-S. Wu · R.-H. Juang $(\bowtie)$

Department of Biochemical Science and Technology, Institute of Microbiology and Biochemistry,

National Taiwan University, AC 2-520, Taipei 106, Taiwan E-mail: juang@ntu.edu.tw

Tel.: + 886-2-33664448

Fax: $+886-2-23631704$

H.-M. Chen

Department of Life Science, Catholic Fu-Jen University, Taipei 242, Taiwan

C.-T. Lin

Institute of Bioscience and Biotechnology,

National Taiwan Ocean University, Keelung 202, Taiwan higher sensitivity to proteolytic modification, but has no change in its kinetic parameters.

Keywords Ipomoea Protein kinase $\cdot$ Protein phosphorylation $\cdot$ Sweet potato roots $\cdot$ Starch phosphorylase

Abbreviations CDPK: Calcium-dependent protein kinase - Glc-1-P: Glucose 1-phosphate - GP: Glycogen phosphorylase - H-SP: High-affinity type SP (Pho2) L-SP: Low-affinity type SP (Pho1) - MBP: myelin basic protein - PVDF: Polyvinylidene fluoride - SP: Starch phosphorylase

\section{Introduction}

Starch phosphorylase (SP, EC 2.4.1.1) catalyzes the reversible phosphorolysis of starch and produces glucose 1-phosphate (Glc-1-P) as one of its products (Hanes 1940a, 1940b). Plants express two isoforms of SP, which are classified as the low-affinity (L-form SP, L-SP or Pho1) and the high-affinity (H-form SP, H-SP or Pho2) types according to their binding affinities to starch (Nakano and Fukui 1986; Nakano et al. 1989; Mori et al. 1991; Albrecht et al. 1998). When compared to the amino acid sequence of glycogen phosphorylase (GP), H-SP shows higher homology to GP. In contrast, a peptide containing 78 amino acids (L78) is inserted in the center of L-SP, where the starch-binding site is located (Mori et al. 1993). The L78 might be derived from an intron (Camirand et al. 1990), and its amino acid sequence contains several interesting features, as predicted by the computer programs, including PEST regions, phosphorylation sites, and a polyproline II helix (Chen et al. 2002). These structural features are summarized in Fig. 1. During the cell division cycle, proteins containing a PEST region are degraded rapidly (Rogers et al. 1986). Indeed, most L-SP molecules isolated from mature potato tubers (Brisson et al. 1989) or sweet potato roots (Chiang et al. 1991) are proteolytically mod- 
ified. Nevertheless, L-SP retains its quaternary structure as well as full catalytic activity. On the SDS-PAGE gel, the purified L-SP reveals an intact $110 \mathrm{kDa}$ band (SP110) and a group of bands (F50s) having a molecular mass of approximately $50 \mathrm{kDa}$, which are produced by the proteolytic modification of L-SP (Chen et al. 2002).

Glycogen phosphorylase from rabbit muscle has complex regulatory mechanisms including the wellknown phosphorylation at the near N-terminal Ser residue by its specific protein kinase (Sprang et al. 1988). On the other hand, SP had never been reported as having any regulation mechanism during the past decades, and its physiological role remains unclear (Nelson and Pan 1995), although pea SP has been reported as inhibited by the physiological concentrations of ADPglucose (Kruger and Ap Rees 1983). However, observations have shown that the accumulation of starch is connected with the increase of SP activity in potato tubers (Mingo-Castel et al. 1976; Albrecht et al. 2001), maize endosperm (Liu and Shannon 1981), rice (Baun et al. 1970), wheat (Schupp and Ziegler 2004), and sweet potato roots (Chang et al. 2000). Starch content is also in proportion to the expression of SP in potato (Brisson et al. 1989; St-Pierre and Brisson 1995; Duwenig et al. 1997; Albrecht et al. 2001), spinach (Duwenig et al. 1997), and pea (Van Berkel et al. 1991). We have reported that the proteolytic modification of L-SP on L78 has a regulatory role for its catalytic behavior (Chen et al. 2002). But how the proteolysis would be conducted and what the upstream regulatory elements would be are still unknown.

Recently, Tetlow et al. (2004) have reported that wheat L-SP is phosphorylated and could form multiprotein complexes with the phosphorylated starch branching enzymes (SBEI and SBEIIb). The computational analysis of the amino acid sequence reveals that several Ser residues on the L78 fragment might be phosphorylated (Chen et al. 2002). In this study, by using the commercial antibodies against phosphoamino acids, we found that L-SP isolated from sweet potato roots is phosphorylated. This led us to search for a protein kinase that is responsible for the phosphorylation of L-SP. Consequently, a protein kinase activity was detected and partially purified that specifically phosphorylated L-SP at one Ser residue on L78. The results in this study imply that the phosphorylation of L78 might play a key role in the regulation of the catalytic features of L-SP in sweet potato roots.

\section{Materials and methods}

\section{Materials and reagents}

Mature roots of sweet potato [Ipomoea batatas (L.) Lam. cv Tainong 57] and potato tubers (Solanum tuberosum L.) were collected from the local farm near Taipei; $\left[\gamma-{ }^{32}\right.$ P]ATP was obtained from NEN Life Sciences, Perkin-Elmer. Mouse monoclonal antibodies against phosphoamino acids (phospho-Ser, phosphoThr, or phospho-Tyr), cellulose phosphate, and protein kinase inhibitor (staurosporine) were from Sigma. The antibody titre has not been determined, and the dilution used for working solutions in immunoblotting followed the directions from Sigma. The antibody against the His tag on the expressed peptides was from Amersham Biosciences (Anti-His antibody). The Polyvinylidene fluoride (PVDF) membrane (Immobilon-P or Immobilon- $\left.\mathrm{P}^{\mathrm{SQ}}\right)$ was purchased from Millipore. Calf intestine alkaline phosphatase was from Boehringer Mannheim. Starch $(1.2 \%$ solution, $\mathrm{w} / \mathrm{v})$ was prepared by dissolving $1.2 \mathrm{~g}$ soluble starch powder (Nacalai Tesque, 321-22, Kyoto, Japan; or Sigma S-9765) in $100 \mathrm{ml}$ warm water $\left(80^{\circ} \mathrm{C}\right)$. Glucose 1-phosphate (Glc-1-P) was purchased from Sigma (G-7000). All other reagents or equipment are specified in the text. Starch phosphorylases (L-SP and $\mathrm{H}-\mathrm{SP}$ ), monoclonal antibody $\mathrm{H} 7 \mathrm{c}$, and monospecific antibody Anti-L2 were prepared as described previously

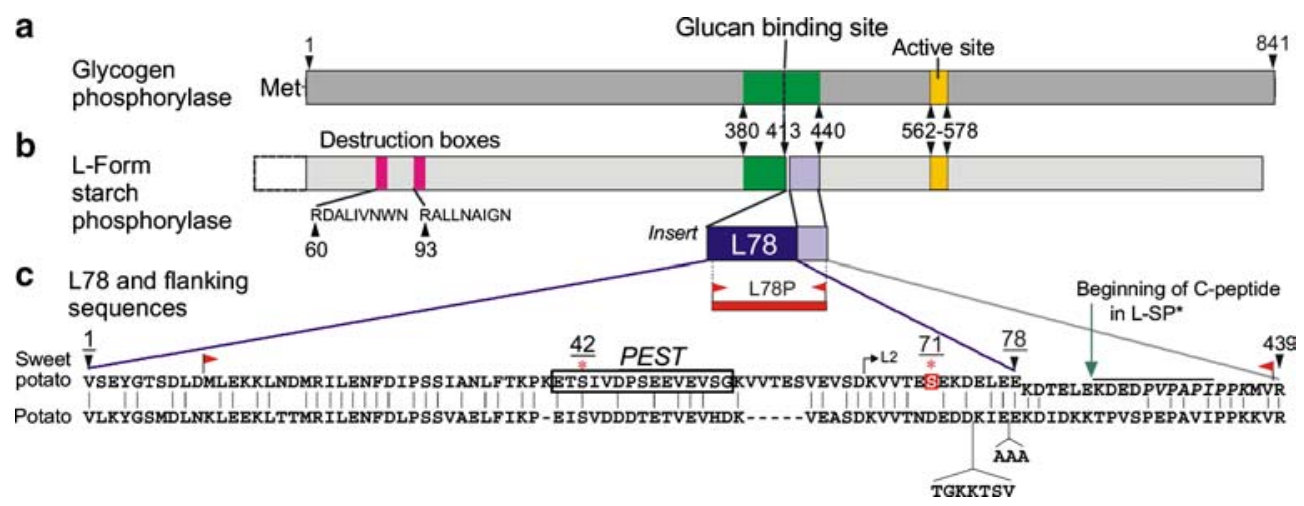

Fig. 1 a-c The structural features of glycogen phosphorylase from rabbit muscle (a) and L-form starch phosphorylase from sweet potato (b) are compared. The L78 insertion on L-SP is emphasized and expanded to show its amino acid sequence (Lin et al. 1991) and significant features (c) including the PEST region and the phosphorylation target for GP kinase. The range of an expressed peptide (L78P) containing the L78 fragment is marked with red triangle flags. The two Ser residues, which were replaced by Ala in site-directed mutants are indicated with asterisks. The amino acid sequence of the L78 insertion from potato (Nakano and Fukui 1986) is aligned, showing 63\% homology with the sweet potato L78 (identical amino acid pairs are marked with a vertical dash) 
(Chen et al. 2002). Phosphorylated L-SP was prepared in vitro by incubating $200 \mu \mathrm{g}$ purified L-SP at $37^{\circ} \mathrm{C}$ in a mixture containing $50 \mathrm{mM}$ HEPES $(\mathrm{pH} 7.0), 5 \mathrm{mM}$ $\mathrm{MgCl}_{2}, 1 \mathrm{mM}$ ATP, protease inhibitor cocktail, and $50 \mu \mathrm{g}$ partially purified kinase from this study. After overnight incubation, the mixture was subjected to $\mathrm{Su}-$ perose $6 \mathrm{HR} 10 / 300$ (Pharmacia) to separate the modified L-SP from LSK and ATP. The fractions containing L-SP were collected and concentrated by ultrafiltration (Microcon YM30, Millipore, Bedford, MA, USA). Protein content was determined by the dye-binding method (Bradford 1976) using the microassay system from Bio-Rad (Protein Assay Kit). Bovine serum albumin was used as the standard.

Polyacrylamide gel electrophoresis, protein transfer, and immunoblotting

The SDS-PAGE was performed according to the method described by Laemmli (1970) with a slight modification. After electrophoresis, the proteins on the gel were transferred to the PVDF membrane that was then stained by antibodies to reveal the target proteins. The detailed protocols are described in our previous report (Chen et al. 2002).

\section{Assays for enzyme activity}

The assays for SP in the synthetic and the phosphorolytic directions were carried out according to the procedures described by Chen et al. (2002) and Mori et al. (1993), respectively.

The kinase activity was assayed by measuring the incorporation of ${ }^{32} \mathrm{P}$ from $\left[\gamma-{ }^{32} \mathrm{P}\right] \mathrm{ATP}$ to $\mathrm{L}-\mathrm{SP}$. The reaction mixture contained $50 \mathrm{mM}$ HEPES $(\mathrm{pH} 7.0)$, $5 \mathrm{mM} \mathrm{MgCl} 2,3.7 \times 10^{4} \mathrm{~Bq}\left[\gamma-{ }^{32} \mathrm{P}\right] \mathrm{ATP}$, and $4 \mu \mathrm{g} \mathrm{L-SP}$ as the substrate. An appropriate amount of the enzyme solution was added to the reaction mixture for a final volume of $10 \mu \mathrm{l}$. This was incubated at $37^{\circ} \mathrm{C}$ for $10 \mathrm{~min}$, terminated by adding to the SDS sample buffer (Laemmli 1970), and then denatured at $95^{\circ} \mathrm{C}$ for $10 \mathrm{~min}$. After gel electrophoresis (12.5\% SDS-PAGE) at $150 \mathrm{~V}$, the gel was stained with Coomassie Brilliant Blue R-250, and was dried by the cellophane sandwich method (Juang et al. 1984). The radioactive bands were exposed on an imaging plate and visualized by an image analyzer (Fujix BAS 1000). Alternatively, the gel was exposed to Amersham Hyperfilm MP directly for a conventional autoradiogram. For quantification of the radioactivity of the phosphorylated proteins, the bands were cut out directly from the gel after the staining and put into the counting vials. After adding the scintillation fluid, the radioactivity was measured in a liquid scintillation counter (Beckman LS5000). The enzyme unit is defined as the femtomole of $\left[\gamma-{ }^{32} \mathrm{P}\right] \mathrm{ATP}$ incorporated into L-SP per minute.
Isolation of L-SP kinase from sweet potato roots

All purification procedures were performed at $4^{\circ} \mathrm{C}$. Fresh sweet potato roots $(150 \mathrm{~g})$ were homogenized in a blender in $150 \mathrm{ml}$ of buffer A $(50 \mathrm{mM}$ Tris- $\mathrm{HCl}$, $\mathrm{pH} 7.4,150 \mathrm{mM} \mathrm{NaCl}$ ) containing $1 \%$ polyvinylpolypyrrolidone (PVPP) and was centrifuged for $20 \mathrm{~min}$ at 21,600 g (Beckman Avanti J25, rotor JLA 16.25). The supernatant was filtered through glass wool and the filtrate collected was then fractionated by ammonium sulfate precipitation at a saturation ranging between 20 and $40 \%$. The precipitated protein was collected by centrifugation as above and was resuspended in a minimal volume of buffer $\mathrm{A}$. The crude protein mixture was dialyzed overnight against buffer A. The precipitate formed was removed by centrifugation $(29,400 \mathrm{~g}$, $40 \mathrm{~min}$ ) before applying it to a DEAE-Sephacel column (i.d. $2.6 \times 20 \mathrm{~cm}$, pre-equilibrated in buffer A). The column was washed with buffer $\mathrm{A}$, and the kinase activity was eluted directly. The active fractions were pooled and then loaded into a phosphocellulose column (total volume $40 \mathrm{ml}$, pre-equilibrated in buffer A). This was eluted by a linear $\mathrm{Na}_{3} \mathrm{PO}_{4}$ gradient $(0$ $200 \mathrm{mM}$ in buffer A, total $150 \mathrm{ml}$ ); the kinase activity appeared at a concentration of approximately $100 \mathrm{mM}$ $\mathrm{Na}_{3} \mathrm{PO}_{4}$. The active fractions were pooled and further purified by gel filtration using Sephacryl S-300 (i.d. $1.6 \times 100 \mathrm{~cm}$, equilibrated in buffer A). The fractions containing kinase were collected and concentrated by ultrafiltration (Amicon YM-30). This purification protocol allowed us to obtain a partially purified kinase, which was used in all the experiments throughout this study.

In-gel kinase assay for L-SP kinase using non-denaturing gel

Isolated kinase was separated by non-denaturing gel electrophoresis in $10 \%$ native polyacrylamide gel containing L-SP $(0.3 \mathrm{mg} / \mathrm{ml})$. After electrophoresis, the gel was equilibrated for $30 \mathrm{~min}$ in a reaction buffer $(50 \mathrm{mM}$ HEPES, $\mathrm{pH} 7.0,5 \mathrm{mM} \mathrm{MgCl} 2$ ). Then it was incubated for $2 \mathrm{~h}$ at $37^{\circ} \mathrm{C}$ in $10 \mathrm{ml}$ of the reaction buffer containing $7.4 \times 10^{5} \mathrm{~Bq}$ of $\left[\gamma-{ }^{32} \mathrm{P}\right] \mathrm{ATP}$. The reaction was terminated by transferring the gel into a bath of $5 \%$ trichloroacetic acid and $1 \% \mathrm{Na}_{3} \mathrm{PO}_{4}$. The unincorporated ATP was removed by washing the gel in the bath solution several times. The radioactive band on the gel was visualized by autoradiography, as described in the assay method for the L-SP kinase activity.

Recombinant L78 DNA preparation and site-directed mutagenesis

The SPc39 (GeneBank accession no. M64362) is a cDNA clone of 3,292 nucleotide residues containing an 
open reading frame encoding for the 955 amino acids of L-SP from sweet potato roots (Lin et al. 1991). According to the SPc39 sequence, a forward primer $\left(5^{\prime}\right.$ GAATTCGGTATCAGAATATGGCACGTC $3^{\prime}$ ) and a reverse primer $\left(5^{\prime}\right.$ CTGACGTGCCATATTCTGATA CCGAATTC 3') were synthesized. A $0.2-\mathrm{kb}$ DNA fragment encoding L78 was amplified using SPc39 as a template. The PCR product was ligated with pCR2.1 and then transformed into Escherichia coli TOPO10 (Invitrogen). The 0.2-kb insert DNA was then subcloned into pET-20b(+) (Novagen) and transformed into $E$. coli BL21 (DE3), which was used for the preparation of the recombinant $\mathrm{L} 78$. The crude protein was purified by a $\mathrm{Ni}^{+}$-nitrilotriacetic acid Sepharose superflow column (Qiagen). The expressed polypeptide (L78P) covers the essential part of L78 and its C-terminal flanking sequence (total 89 amino acids), which is shown in Fig. 1c. $\mathrm{L} 78 \mathrm{P}$ was used to immunize $\mathrm{BALB} / \mathrm{c}$ mice and produce hybridoma secreting monoclonal antibody ( $\mathrm{mAb}$ antiL78P) following the protocol described previously (Chen et al. 2002).

The recombinant L78 DNA in pET-20b(+) prepared above was used as the wild-type template for site-directed mutagenesis using the QuikChange Site-Directed Mutagenesis Kit (Stratagene). Three mutants at residues Ser42 or/and Ser71 were prepared, and their sequences were verified by nucleotide sequencing. The expressed proteins [L78P(S42A), L78P(S71A), and L78P(S42A/ S71A)] were isolated and purified by affinity chromatography as described above.

Analysis for phosphoamino acids on the phosphorylated L-SP

Free phosphoamino acids can be separated and analyzed by a two-dimensional thin-layer electrophoresis system (Boyle et al. 1991). The radioactive labeled proteins, as described in the assay method for the L-SP kinase, were separated by $12.5 \%$ SDS-PAGE and then transferred to the PVDF membranes. The radioactive bands were excised, hydrolyzed in $200 \mu \mathrm{l}$ of $5.7 \mathrm{~N} \mathrm{HCl}$ for $1 \mathrm{~h}$ at $110^{\circ} \mathrm{C}$, and then dried to a powder form in a vacuum microcentrifuge. Five microliters of the first-dimension buffer $(0.58 \mathrm{M}$ formic acid, $1.36 \mathrm{M}$ glacial acetic acid, $\mathrm{pH}$ 1.9) containing the mixture of phosphoamino acid standards (Sigma, P-Ser, P0878; P-Thr, P1053; P-Tyr, P9405; $1.7 \mu \mathrm{g} / \mu \mathrm{l}$, respectively) was added to dissolve the powder, which was then applied as a single spot to the DC-Platten cellulose thin-layer plate (Merck 1.05716, $20 \times 20 \mathrm{~cm})$. After wetting the plate with the firstdimension buffer, electrophoresis was performed for $20 \mathrm{~min}$ at $1,500 \mathrm{~V}$. The plate was dried completely, and then the second dimension was performed for $16 \mathrm{~min}$ at $1,300 \mathrm{~V}$ (second-dimension buffer: $0.5 \%$ pyridine, $0.87 \mathrm{M}$ glacial acetic acid, $\mathrm{pH}$ 3.5). Phosphoamino acid standards were visualized by spraying the plate with $0.25 \%(\mathrm{w} / \mathrm{v})$ ninhydrin in methanol and heating it at $65^{\circ} \mathrm{C}$ for $30 \mathrm{~min}$. For the radioactive labeled amino acids, the X-ray film was exposed to the plate for $1 \mathrm{~d}$ at $-80^{\circ} \mathrm{C}$.

a

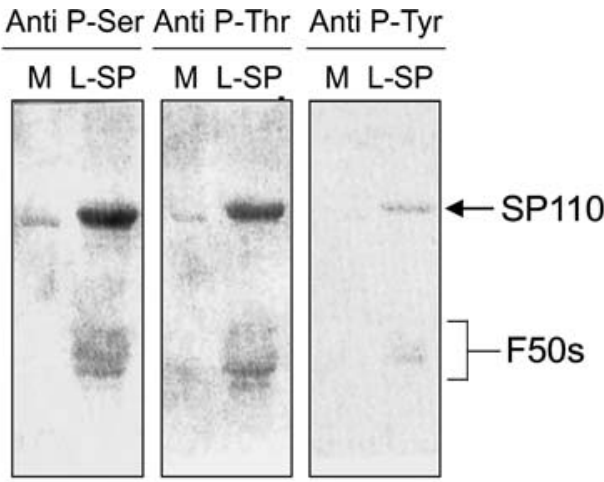

b

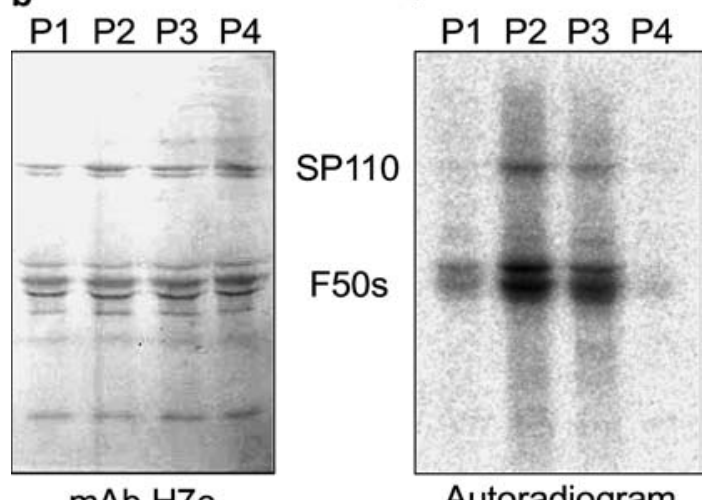

C

$\mathrm{mAb} \mathrm{H7c}$

Autoradiogram

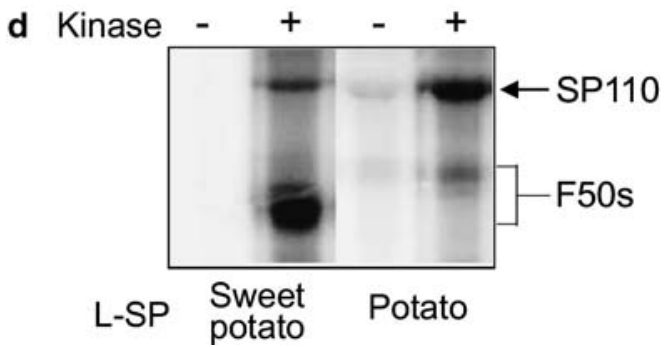

Fig. 2 a Purified L-SP ( $5 \mu \mathrm{g}$ per lane) was analyzed by monoclonal antibodies against phospho-Ser $(P-S e r)$, phospho-Thr $(P-T h r)$, or phospho-Tyr $(P-T y r)$. Molecular weight marker $(M)$ from Pharmacia (LMW electrophoresis calibration kit) was used as the negative control for immunoblotting. One of the markers (phosphorylase b) was stained slightly. b and $\mathbf{c}$ L-SP was phosphorylated in vitro by protein fractions from sweet potato roots using radioactive ATP. Ammonium sulfate fractions of the crude extract at $0-20 \%(P 1), 20-40 \%(P 2), 40-60 \%(P 3)$, and $60-80 \%(P 4)$ saturation were used as the source of the protein kinase. After the enzyme reaction, the L-SP was pulled down by a mixture of Protein A-Sepharose and monoclonal antibody H7c (specific to L-SP). The precipitate was analyzed by SDS-PAGE and then transfered to a membrane. Antibody H7c was used again to reveal L-SP bands on the blot (b), which was then exposed on a phospho-image plate, and visualized on the autoradiogram (c). The kinase isolated from sweet potato roots also phosphorylated L-SP from potato tubers (d, $6 \mu \mathrm{g}$ protein per lane) 


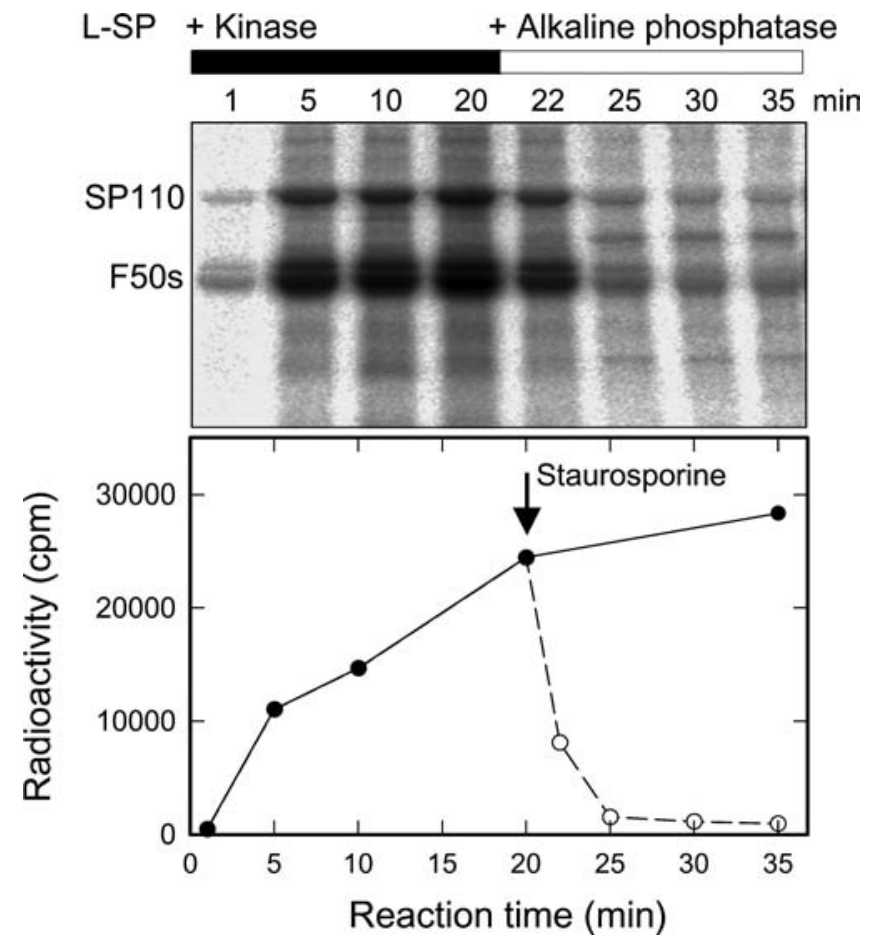

Fig. 3 The phosphorylation of L-SP by the protein kinase (closed circles) and the subsequent dephosphorylation by calf intestine alkaline phosphatase (open circles) were monitored during the reaction. Partially purified kinase $(2.8 \mu \mathrm{g})$ was added to $50 \mu \mathrm{g}$ of LSP in a total volume of $100 \mu$ l. Phosphorylation was terminated after 20 min by adding the kinase inhibitor staurosporine $(1 \mu \mathrm{g} / \mu \mathrm{l}$, indicated by an arrow), and then the calf intestine alkaline phosphatase (five units) was added to remove the phosphate group on the protein. The autoradiogram shows the accumulation and disappearance of the phosphorylated bands at selected time points (10 $\mu \mathrm{l}$ aliquot was sampled for each lane). One aliquot of the original phosphorylation mixture was kept to complete the whole reaction after the addition of staurosporine (closed circle at $35 \mathrm{~min}$ )

\section{Results}

The L-SP is found phosphorylated in sweet potato roots or phosphorylated in vitro by a protein fraction from the root extract

The L-SP isolated from the sweet potato roots was separated on SDS-PAGE, transferred to the PVDF membrane and then probed with commercial antibodies against phosphoamino acids. The immunoblotting results (Fig. 2a) showed that L-SP in the sweet potato roots is phosphorylated intensively on its Ser or Thr residues, but only faintly on Tyr. Both the intact L-SP (SP110) and the protease-cleaved fragments of L-SP (F50s) are phosphorylated, but the SP110 bands show a higher intensity of staining, indicating that the intact LSP might be phosphorylated more extensively than the protease-cleaved form in the sweet potato, or the $110 \mathrm{kDa}$ protein is more abundant in the roots. To further verify the phosphorylation of L-SP, we performed the cell-free reaction by mixing fractions of ammonium sulfate precipitation from the crude extract of sweet potato roots with the purified L-SP as the substrate. The fraction of 20 to $40 \%$ ammonium sulfate saturation showed the highest phosphorylation activity for L-SP (Fig. 2c). In contrast to the phosphorylation detected by monoclonal antibodies (Fig. 2a), the proteolytic modified L-SP (F50s) is phosphorylated more intensively in the in vitro reaction. In addition to the LSP from sweet potato, the kinase also phosphorylates L-SP from potato (Fig. 2d). Notably, the sweet potato L-SP was phosphorylated more intensively on its F50s fragments, whereas the intact SP110 was the major phosphorylation target in the potato L-SP (Fig. 2d). In Fig 3, the L-SP was phosphorylated by the kinase, stopped by staurosporine, and then the phosphorylation was reversed by calf intestine alkaline phosphatase, which removed most of the labeled radioactive phosphate from L-SP. The above results indicate the existence of a protein kinase in sweet potato roots, which might phosphorylate L-SP on its Ser or Thr residues.

A protein kinase is partially purified from sweet potato roots

The kinase activity in the sweet potato roots was traced by using L-SP as the substrate in a purification protocol. A putative L-SP kinase was partially purified by conventional liquid chromatography (Fig. 4) starting from the fraction of 20 to $40 \%$ saturation of ammonium sulfate. The major kinase activity flowed through the DEAE ion exchange column directly and split into two peaks on the chromatogram (Fig. 4a). We repeated this operation several times and confirmed that these two peaks are actually from one larger peak, whose activity is affected by an unknown factor eluted coincidently. The final purification table (Table 1) shows an increasing specific activity through the whole purification protocol except in the cellulose phosphate step. This is due to the inhibitory effect of the phosphate used in the gradient elution for the adsorbed proteins. Nevertheless, the activity could be fully recovered by removing the phosphate from the sample in the next gel filtration step. Totally, the enzyme was purified by 500 -fold and $36 \%$ activity was recovered. Its native molecular mass was determined as $338 \mathrm{kDa}$ by gel filtration (Fig. 4d). This partially purified kinase was visualized by the in-gel kinase assay using non-denaturing PAGE that contained L-SP as the substrate in the gel (Fig. 4d, inset).

\section{L-SP is phosphorylated specifically on a Ser residue} of $\mathrm{L} 78$

To examine the substrate specificity of the kinase, partially purified H-SP, the extensively proteolytic modified L-SP (L-SP*), and an expressed peptide (L78P) containing L78 were used as the alternative substrates for comparison. H-SP is the high-affinity isoform of SP, which shows higher homology to GP. It contains no L78 

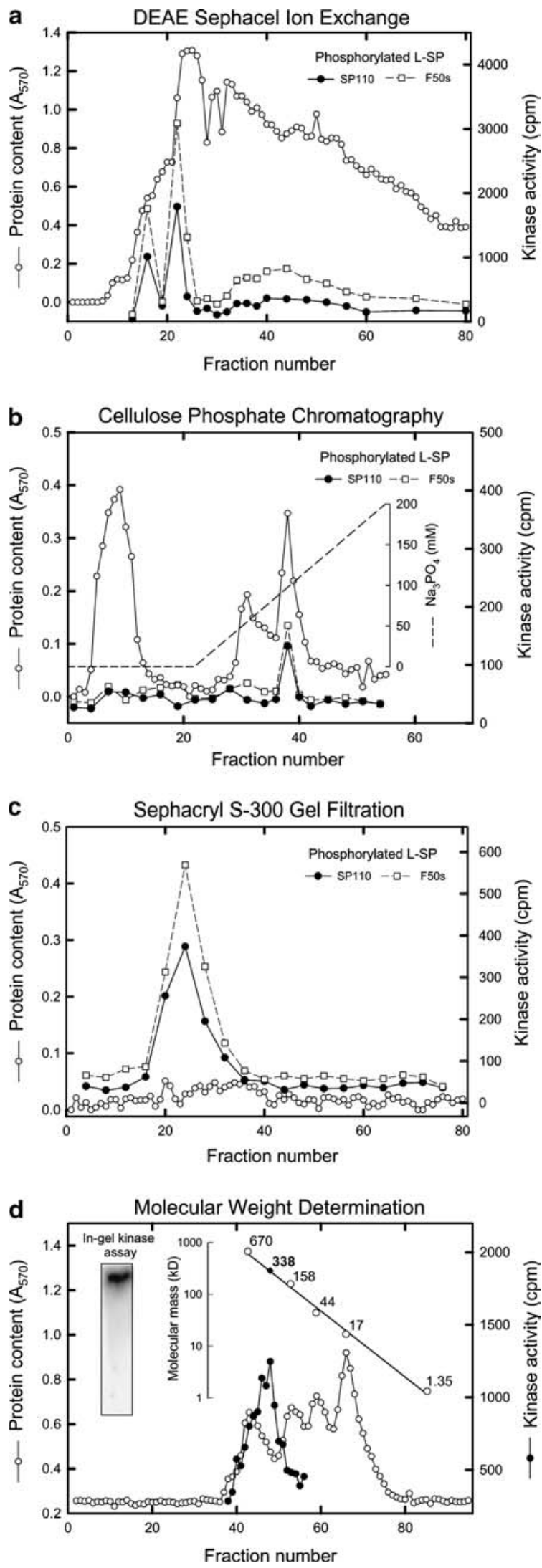

in its molecule and has a smaller molecular mass of $97 \mathrm{kDa}$ (Mori et al. 1993). L-SP* was prepared by removing the L78 fragment from L-SP by controlled
Fig. 4 The kinase was isolated by ammonium sulfate fractionation first and then subjected to liquid chromatography using DEAE Sephacel (a), cellulose phosphate (b), and Sephacryl S-300 (c). Kinase activity was determined by measuring the radioactivity of the L-SP bands on SDS-PAGE gel. Since L-SP was resolved into the intact subunit (SP110) and a group of fragments with molecular mass around $50 \mathrm{kDa}$ (F50s), their radioactivity was measured separately (SP110, close circles; F50s, open squares). The molecular mass of the putative L-SP kinase was estimated as $338 \mathrm{kDa}$ by gel filtration using Sephacryl S-300 (d). The molecular weight markers (Bio-Rad, 151-1901) are thyroglobulin $(670 \mathrm{kDa})$, bovine gamma globulin $(158 \mathrm{kDa})$, chicken ovalbumin (44 kDa), equine myoglobin $(17 \mathrm{kDa})$, and vitamin B12 (1,350 Da). The kinase eluted from the gel filtration was collected and concentrated, and then a fraction of $2 \mu \mathrm{g}$ protein was subjected to the in-gel kinase assay (inset in d). Representative data shown $(n=6)$

proteolysis (Chen et al. 2002). Figure 5 shows that no phosphorylation was found on H-SP and L-SP*. On the other hand, the kinase intensively phosphorylated the expressed peptide L78P that contains the L78 fragment.

To determine the amino acid that was phosphorylated by this kinase, protein substrates including L-SP, L78P and myelin basic protein (MBP) were phosphorylated by using radioactive ATP. The phosphorylated proteins were separated by SDS-PAGE and transferred to the PVDF membrane, which were then cut out for analysis. SP110 and F50s were collected separately for L-SP. They were then subjected to acid hydrolysis and separated by two-dimensional electrophoresis as described in the experimental procedures. Figure 6 reveals that phospho-Ser is the major phosphoamino acid in all the cases. A trace amount of phospho-Thr was observed in SP110, F50s, and MBP. Notably, L78P shows only phospho-Ser. The above observations imply that L-SP is the specific target for the kinase that could phosphorylate Ser residue(s) on $\mathrm{L} 78$.

The exact phosphorylation site on L-SP was determined by using mutants of L78P by site-directed mutagenesis, in which two key Ser residues were replaced by Ala: L78P(S42A), L78P(S71A), and L78P(S42A/S71A). The two residues were selected because $S 42$ is flanked by a predicted sequence of GP kinase target (KETSI) (Chan et al. 1982), and Ser71 was predicted as the strongest phosphorylated site on L78 (Chen et al. 2002). The amino acid sequence of L78 is aligned in Fig. 1c, which is numbered from 1 to 78 using underlined numbers (in the total L-SP molecule, L78 starts from Val 414 as the numbering system of rabbit GP is employed). Figure 7 shows that wild-type L78P and L78P(S42A) are phosphorylated intensively, but mutation at Ser71 totally suppresses the phosphorylation. This result suggests that Ser71 is the only residue being phosphorylated on L78.

The phosphorylated L-SP is sensitive to proteolytic modification, but has no change in its kinetic parameters

The crude extract from the sweet potato roots was mixed with the in vitro phosphorylated L-SP and incubated to 

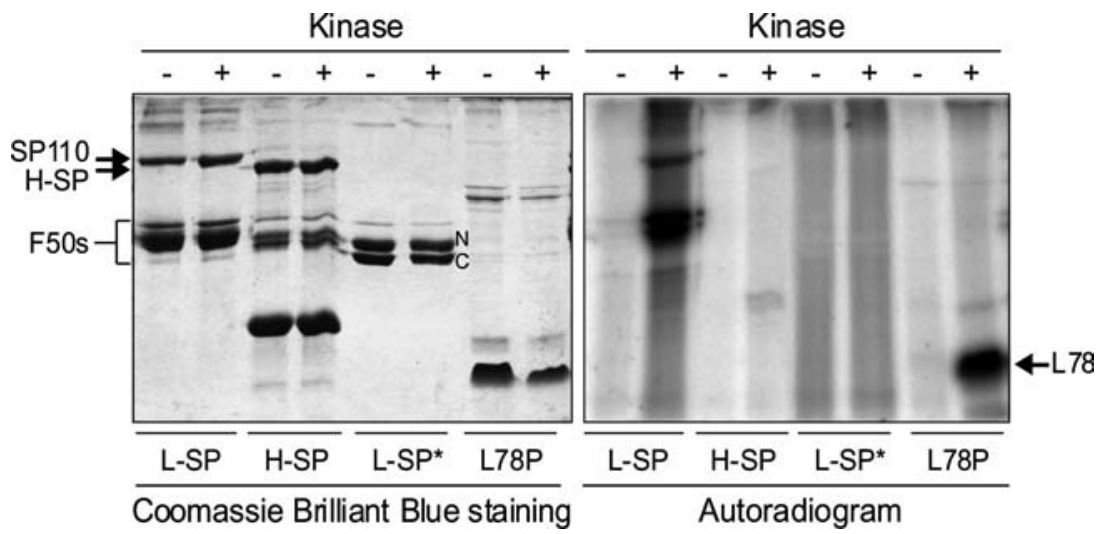

Fig. 5 The catalytic specificity of the kinase was examined on SDS-PAGE gel (12.5\%) by using L-SP and H-SP as the substrates. Modified protein $\left(L-S P^{*}\right)$ or expressed peptide derived from L-SP $(L 78 P)$ were used for comparison. Neither H-SP nor L-SP* could be phosphorylated. L-SP* contained two large peptides after the controlled proteolytic modification on L-SP (denoted with $N$ and $C$ ), which have been identified by amino acid sequencing. The $N$-terminal sequence of the $C$-peptide was determined as KDEDPVPAPI that is indicated in Fig. 1c. The H-SP used in this experiment was not homogeneous, but the 97-kDa band on the gel has been identified as H-SP by tryptic digestion and mass spectroscopic analysis

observe the effect of phosphorylation on L-SP. It was found that the phosphorylated enzyme was degraded after $12 \mathrm{~h}$ of incubation (Fig. 8). The intact SP110 disappeared completely, and the F50s showed an increase of its electrophoretic mobility on SDS-PAGE gel as revealed by using $\mathrm{mAb} J 3 \mathrm{~b}$, which recognizes an epitope on the N-terminal side of the protein (Fig. 8b). On the other hand, $\mathrm{mAb}$ anti-L78P was prepared by using L78P as the antigen, and it might recognize a specific epitope on the L78 of L-SP. If anti-L78P was used instead of $\mathrm{mAb} \mathrm{J3b}$, a distinct contrast was observed (Fig. 8c). Anti-L78P cannot stain any band on the blot after $15 \mathrm{~h}$, indicating that the L78 insertion might be removed completely during the proteolytic modification induced by the phosphorylation of L-SP; otherwise the unmodified L-SP is unaffected by the crude extract throughout the incubation (left panels in Fig. 8). Apparently, the crude extract might contain an unidentified proteolytic system, which could specifically recognize the phosphorylated form of L-SP and cleave the enzyme on its L78 insertion. Further study is needed to elucidate this effect.

The phosphorylation did not significantly affect the kinetic parameters $\left(K_{\mathrm{m}}\right.$ or $\left.K_{\mathrm{cat}}\right)$ of L-SP, either in the direction of phosphorolysis or amylose synthesis (Table 2). The effect of phosphorylation on L-SP is therefore very different from the phosphorylation on the Ser14 of GP, which triggers the change of protein conformation and thereby activates the enzyme (Sprang et al. 1988). Interestingly, the ratio of the $K_{\text {cat }}$ in the synthetic direction over the phosphorolytic direction is about five. This observation correlates with our observation that when Glc-1-P is used as the exclusive substrate and catalyzed by L-SP for the synthesis of amylose, the polymerization reaction achieved an equilibrium state where the ratio of the product $\left(\mathrm{P}_{\mathrm{i}}\right)$ to the
Fig. 6 Several substrate proteins (L-SP, L78P, and MBP, $5 \mu$ g each) were phosphorylated using $\left[\gamma-{ }^{32} \mathrm{P}\right] \mathrm{ATP}$, and then the phosphoamino acids were analyzed by two-dimensional thin-layer electrophoresis. Results show that Ser is the primary phosphorylation target on L-SP. $S$ phospho-Ser, $T$ phospho-Thr, $P_{\mathrm{i}}$ inorganic phosphate, " $x$ " marks the origin spot of the sample, and the asterisks denote phosphopeptides by partial hydrolysis

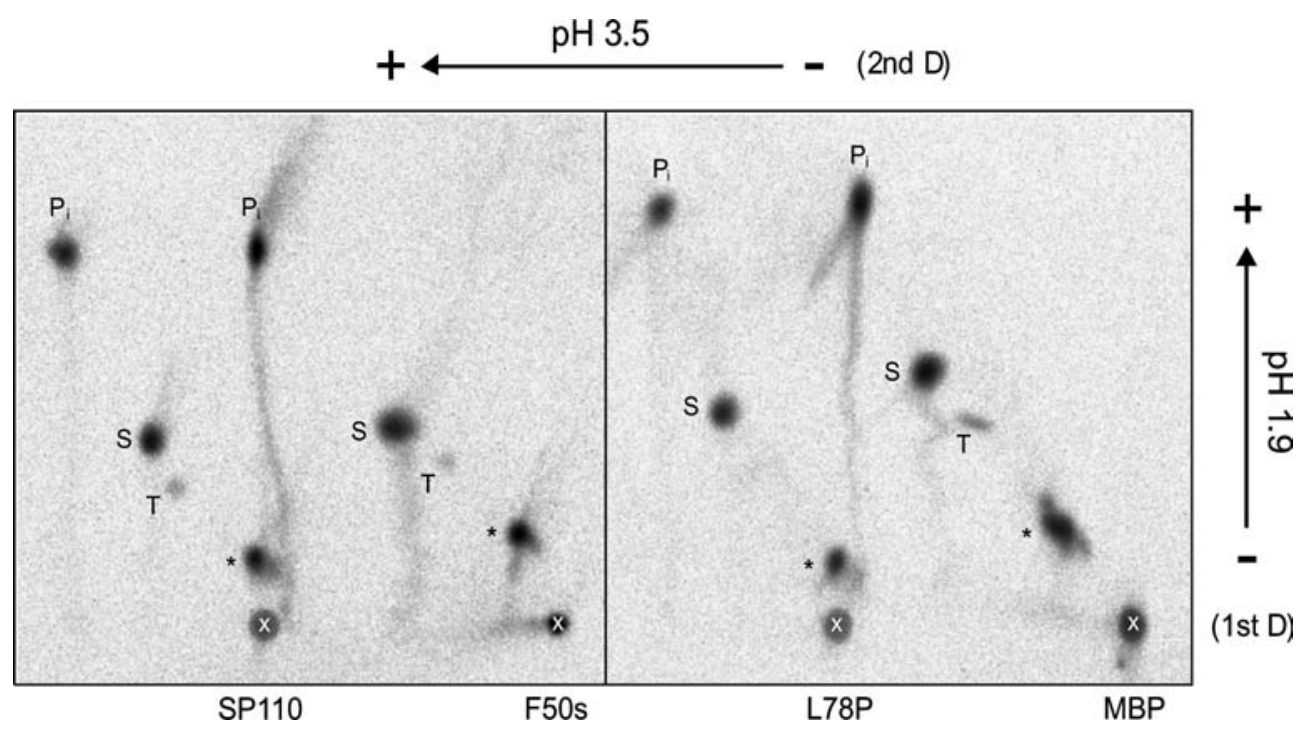



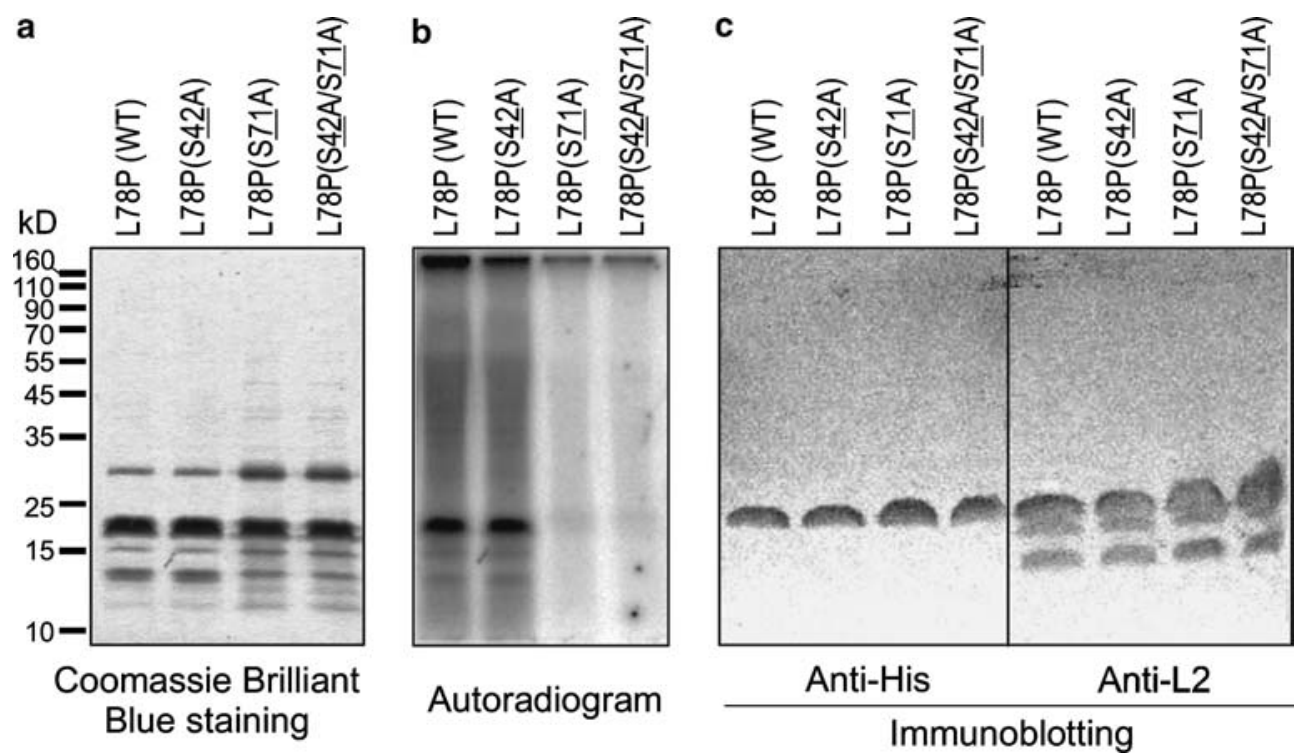

Fig. 7 a-d Mutants of L78P by site-directed mutagenesis replacing Ser residue(s) with Ala were constructed. The expressed peptides ( $3 \mu \mathrm{g}$ per lane) were phosphorylated by the kinase using $\left[\gamma-{ }^{32} \mathrm{P}\right]$ ATP and then separated on SDS-PAGE gel, which was stained with Coomassie Brilliant Blue (a), or transferred to the PVDF membrane and then detected by Anti-His antibody (c) or monospecific antibody Anti-L2 (d). Anti-L2 was prepared by immunizing mice with a synthetic peptide L2, which contained 23 amino acids starting from Lys66 on L78. The Coomassie Brilliant Blue stained gel was dried and then exposed on an imaging plate to visualize the phosphorylated peptides (b). No phosphorylation was observed on mutants whose Ser71 residues were replaced by Ala. The two blots (c and d) look like they have been mistakenly placed upside down; after a careful examination and duplicate experiment, the bands were proved to be stained like that. This might be caused by the small molecular mass of L78P, which contains a high percentage of charged amino acids, and a relatively high concentration of the SDS gel $(17.5 \%)$

remaining substrate (Glc-1-P) was approximately five (data not shown).

\section{Discussion}

In this study, we show that L-SP isolated from sweet potato roots is phosphorylated, as observed by using antibodies against phosphoamino acids (Fig. 2a). The enzymatic phosphorylation activity was further reconstituted in vitro by mixing the protein fractions extracted from sweet potato roots with the purified L-SP (Fig. 2c). In a time-course study, the kinase activity was terminated by the protein kinase inhibitor staurosporine, and the phosphorylated L-SP was then dephosphorylated by the alkaline phosphatase from the calf intestine (Fig. 3). Therefore, we conclude that a portion of L-SP has been phosphorylated in the cell before it is isolated from sweet potato roots, and a protein kinase activity is in existence in the root extract. On the other hand, some isolated LSP might remain unphosphorylated and could be labeled with radioactive phosphate in the test tube. This is reflected in the distinct patterns of intensity between the immunoblot (Fig. 2a) and the autoradiogram (Fig. 2c). However, unidentified protein phosphatase might be present in the sweet potato roots, and the phosphate group of the phosphorylated L-SP could be removed during the extraction procedure for L-SP. We then isolated this kinase (Fig. 4 and Table 1), which could be visualized by the in-gel kinase assay using a non-dena- turing gel, and showed its low mobility on the gel (inset in Fig. 4d), which might be caused by the high affinity between the kinase and L-SP. However, we were not able to visualize a clear protein band of the kinase on SDS-PAGE gel owing to the low abundance of the enzyme in the cell. Besides, the in-gel assay for the kinase activity in SDS-PAGE was not successful. This may be due to the nature of the L-SP structure, whose conformation might be sensitive to SDS.

If the L78 was removed from L-SP by a controlled proteolytic modification, the remaining peptide chains still keep the intact quaternary structure and full catalytic activity (Chen et al. 2002). However, this modified L-SP could not be phosphorylated by the kinase (L-SP* in Fig. 5). Similarly, the H-SP isoform contained no L78 when it was biosynthesized and was not phosphorylated. Therefore, we might regard this kinase as specific to the L78 insertion on the L-SP molecule, although it also phosphorylated MBP, which is a broad-spectrum substrate for most protein kinases (Fig. 6). There are ten Ser and five Thr residues on L78. Analysis for the hydrolysate of the phosphorylated proteins showed that L-SP was intensively phosphorylated on Ser and slightly on Thr residue(s) (Fig. 6). This result supports the observation of using antibodies against phospho-amino acids (Fig. 2a) and is consistent with the observation for the phosphorylation of L-SP in wheat (Tetlow et al. 2004). The exact phosphorylation site was further determined by single amino acid substitutions at two Ser residues on L78. To our surprise, only Ser71 was phosphorylated, 
Fig. 8 a-c The phosphorylated L-SP was sensitive to proteolytic modification as compared with the unmodified L-SP in the reconstitute reaction. Fresh crude extract from the sweet potato roots was mixed with the L-SP

(unmodified or

phosphorylated), and their final protein concentrations were adjusted to $1.5 \mu \mathrm{g} / \mu \mathrm{l}$. The reactions were incubated at $37^{\circ} \mathrm{C}$ for $24 \mathrm{~h}$. Samples of $15 \mu \mathrm{l}$ aliquot from the reaction mixture were collected every $3 \mathrm{~h}$ and then subjected to $12.5 \%$ SDS-PAGE. The gels were either stained with Coomassie Brilliant Blue (a) or transferred to PVDF membranes that were then stained with monoclonal antibodies J3b (b) or anti-L78P (c)

\section{Coomassie Brilliant Blue R staining}

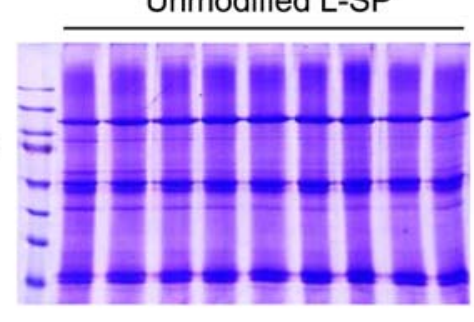

Unmodified L-SP

b

$\mathrm{mAb} J 3 b$
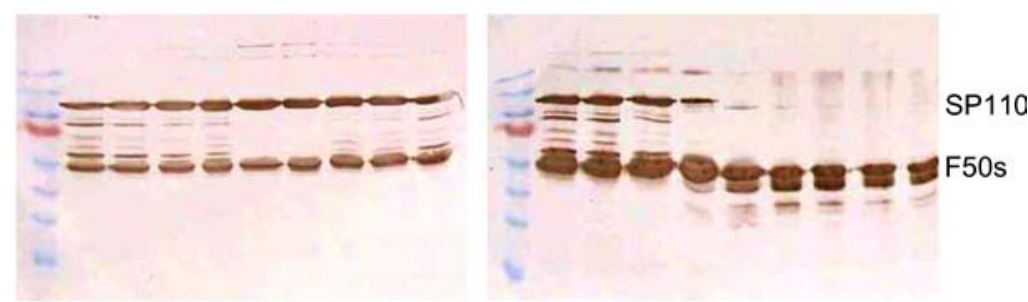

C

$\mathrm{mAb}$ anti-L78P

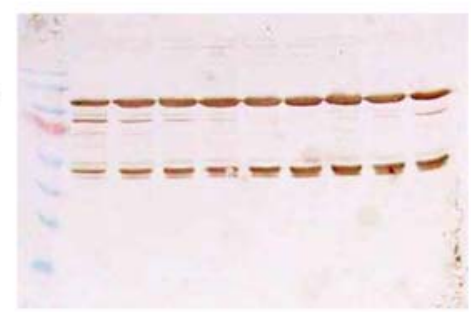

M $\quad 0 \quad 3 \quad 6 \quad 91215182124$
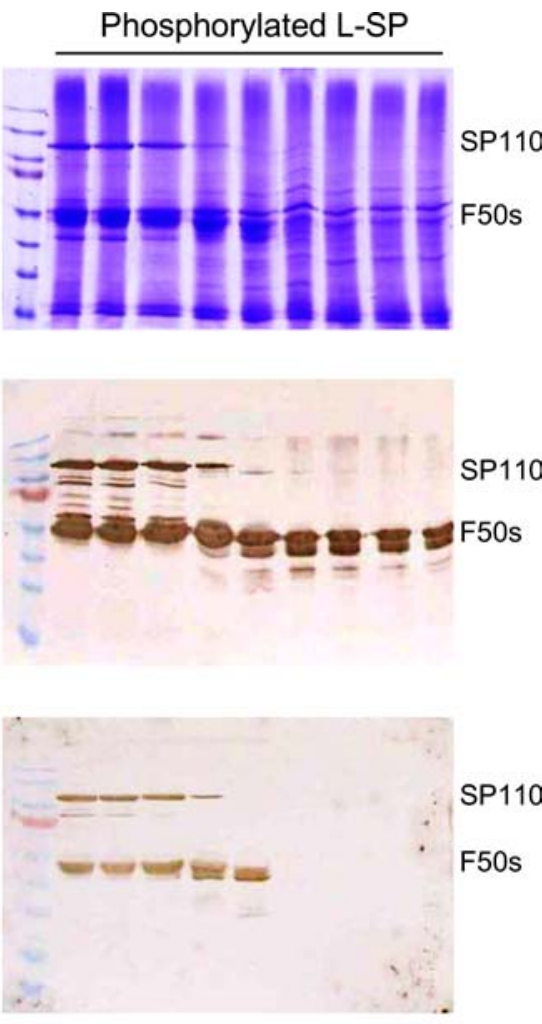

M $030 \quad 6 \quad 91215182124$ (h) and Ser42 was not a target (Fig. 7). Notably, Ser42 is covered in the PEST sequence, as well as a consensus sequence (KXXSI, Fig. 1c), which was predicted as a specific target for GP kinase in mammals (Chan et al. 1982). The GP kinase from rabbit muscle is classified as a member of the calcium-dependent protein kinase (CDPK) subfamily (Hardie 1999). Several CDPK type kinases have been found in plants. For example, sucrose synthase from maize leaf is phosphorylated by a CDPK, and the $K_{\mathrm{m}}$ values for its substrates are reduced by fivefold (Huber et al. 1996). The family class of the kinase in this study is unknown at present, which could be predicted from the amino acid sequence deciphered from its cDNA sequence.

The L-SP (Phola) from potato tubers also contains the L78 insertion, and it could be phosphorylated by the kinase isolated from sweet potato roots (Fig. 2d). These two plant enzymes share high homology in amino acid sequences ( $88 \%$ similarity and $84 \%$ identity). However, sequences of their L78 insertions show only $63 \%$ identity as compared in Fig. 1c. In addition, the corresponding Ser71 position on the potato L78 is replaced by an Asp. The Ser 42 residue is conserved on potato L78, but the KXXSI sequence flanking Ser42 has been changed (-XXSV) in which the Lys residue is deleted, and the Ile is replaced by Val. This suggests that potato L-SP might be phosphorylated by an alternative mechanism. The exact site for phosphorylation on the potato L-SP remains to be clarified.

The results allow us to conclude that L-form SP is site-specifically phosphorylated by a protein kinase in plants; what physiological role might it play? First, one possible effect is the modification of its catalytic behavior due to the conformational change induced by phosphorylation. Our observations on the kinetic parameters for the phosphorylated L-SP decline this

Table 1 The purification of the L-SP kinase from sweet potato roots

\begin{tabular}{|c|c|c|c|c|c|}
\hline Purification steps & $\begin{array}{l}\text { Total activity } \\
\text { (units) }\end{array}$ & $\begin{array}{l}\text { Total protein } \\
(\mathrm{mg})\end{array}$ & $\begin{array}{l}\text { Specific activity } \\
\text { (units } \mathrm{mg}^{-1} \text { ) }\end{array}$ & $\begin{array}{l}\text { Fold } \\
\text { purification }\end{array}$ & $\begin{array}{l}\text { Activity } \\
\text { recovered }\end{array}$ \\
\hline Crude extraction & 342 & 1,845 & 0.19 & 1.0 & 100 \\
\hline $\begin{array}{l}\text { Ammonium sulfate fractionation } \\
(20-40 \% \text { saturation })\end{array}$ & 418 & 835 & 0.50 & 2.6 & 122 \\
\hline DEAE Sephacel & 236 & 105 & 2.25 & 12.0 & 69 \\
\hline Sephacryl S-300 & 123 & 1.3 & 95 & 500 & 36 \\
\hline
\end{tabular}

Purification was started from $150 \mathrm{~g}$ of fresh sweet potato roots (about $5 \mathrm{~cm}$ in diameter)

One enzyme unit is defined as one fmole of $\left[\gamma-{ }^{32}\right.$ P]ATP incorporated in L-SP per minute 
Table 2 Enzyme kinetic analysis of the L-SP and its phosphorylated form

\begin{tabular}{|c|c|c|c|c|}
\hline \multirow[t]{2}{*}{ L-SP } & \multicolumn{4}{|c|}{ Synthetic direction (Chen et al. 2002) } \\
\hline & Soluble starch $(\%, w / v)$ & Glc-1-P (mM) & Fixed $[\text { Glc-1-P] }]^{\mathrm{a}}$ & Fixed [soluble starch] $]^{\mathrm{b}}$ \\
\hline Unmodified & 0.077 & 1.052 & 100.1 & 99.2 \\
\hline Phosphorylated & 0.070 & 1.090 & 98.6 & 97.1 \\
\hline
\end{tabular}

Phosphorolytic direction (Mori et al. 1993)

\begin{tabular}{|c|c|c|c|c|}
\hline & \multicolumn{2}{|l|}{$K_{\mathrm{m}}$} & \multicolumn{2}{|l|}{$K_{\text {cat }}$} \\
\hline & Soluble starch $(\%, w / v)$ & $\mathrm{P}_{\mathrm{i}}(\mathrm{mM})$ & Fixed $\left[\mathrm{P}_{\mathrm{i}}\right]^{\mathrm{c}}$ & Fixed [soluble starch $]^{\mathrm{d}}$ \\
\hline Unmodified & 0.115 & 1.498 & 22.39 & 18.46 \\
\hline Phosphorylated & 0.108 & 1.443 & 21.92 & 18.06 \\
\hline
\end{tabular}

Data were taken from the average of three experiments

a $[$ Glc-1-P $]=4 \mathrm{mM}$

b [soluble starch] $=0.3 \%$

${ }^{\mathrm{c}}\left[\mathrm{P}_{\mathrm{i}}\right]=5 \mathrm{mM}$

$\mathrm{d}$ [soluble starch] $=0.2 \%$

possibility (Table 2). Second, site-specific phosphorylation was reported to target the inhibitor of a transcription factor $(\mathrm{I} \kappa \mathrm{B} \alpha)$ to the ubiquitin-proteasome pathway (Chen et al. 1996), which leads to the proteolytic modification of the protein. Our previous work has reported that the proteolytic modification of the L78 on L-SP might regulate the catalytic direction of the enzyme (Chen et al. 2002). Besides, two destruction boxes (RXXLXXXXN) near the N-terminus were identified on the L-SP from sweet potato as shown in Fig. $1 \mathrm{~b}$. Protein with destruction box has been reported to be degraded through the ubiquitin-dependent proteolysis (Glotzer et al. 1991). Indeed, we have detected proteasome in the crude extract of mature sweet potato roots, which might be associated with L-SP (Chang et al. 2000). Furthermore, the reconstitute experiment that mixed the in vitro phosphorylated L-SP with the crude extract from sweet potato roots shows that phosphorylation might direct the enzyme to the proteolytic modification, which removes the L78 from the L-SP completely (Fig. 8). Third, the recent report by Zeeman et al. (2004) suggests that plastidial $\alpha$-glucan phosphorylase is not required for starch degradation but plays a role in the tolerance of abiotic stress in Arabidopsis leaves. Tetlow et al. (2004) showed the phosphorylation of SP in wheat and the formation of multiprotein complexes with other enzymes related to starch biosynthesis. These observations suggest a wider role for the phosphorylation of L-SP in the control of starch metabolism. To our knowledge, L-SP is the only enzyme catalyzing the synthesis of a glucan from monosaccharide (Glc-1-P) in the absence of a pre-existing primer (data not shown). Moreover, Albrecht et al. (2001) have reported that an antibody specific to the L78 insertion on the potato functional dimer of L-SP (Phola) ${ }_{2}$ almost completely impedes the capacity for the unprimed glucan synthesis of L-SP. Their finding implies that L78 might involve itself in the biosynthesis of starch. The above observations might suggest a key role for the phosphorylation of L78 in the regulation of L-SP catalytic activity through the proteolytic pathway, which might subsequently involve in controlling the regulatory network of starch metabolism in plants. Observations in this study propose a possible role for the L-SP and its phosphorylation, which does not infringe the current view of starch biosynthesis in plants (Ball et al. 1996).

Acknowledgments We thank Drs. D.S. Bendall and B.G. SchlarbRidley (University of Cambridge); Dr. S.C. Chang (Harvard Medical School); Drs. Y.Y. Charng and C.S. Chen (Academia Sinica, ROC); Dr. C.M. Chiang (Case Western Reserve University); Drs. P.L. Huang, A.Y. Wang, and C.C. Yang (National Taiwan University); and Dr. A.B. Tobin (University of Leicester) for their helpful suggestions and discussions. We also thank Dr. J. Hook for reading the article. Part of this work was supported by the National Science Council, ROC.

\section{References}

Albrecht T, Greve B, Pusch K, Kossmann J, Buchner P, Wobus U, Steup M (1998) Homodimers and heterodimers of Pho1-type phosphorylase isoforms in Solanum tuberosum $\mathrm{L}$ as revealed by sequence-specific antibodies. Eur J Biochem 251:343-352

Albrecht T, Koch A, Lode A, Greve B, Schneider-Mergener J, Steup M (2001) Plastidic (Pho1-type) phosphorylase isoforms in potato (Solanum tuberosum L.) plants: expression analysis and immunochemical characterization. Planta 213:602-613

Ball S, Guan HP, James M, Myers A, Keeling P, Mouille G, Buleon A, Colonna P, Preiss J (1996) From glycogen to amylopectin - a model for the biogenesis of the plant starch granule. Cell 86:349-352

Baun CC, Palmiano EP, Perez CM, Juliano BO (1970) Enzymes of starch metabolism in the developing rice grain. Plant Physiol 46:429-434

Boyle WJ, Van der Geer P, Hunter T (1991) Phosphopeptide mapping and phosphoamino acid analysis by two-dimensional separation on thin-layer cellulose plates. Methods Enzymol 201:110-149 
Bradford MM (1976) A rapid and sensitive method for the quantitation of microgram quantities of protein utilizing the principle of protein-dye binding. Anal Biochem 72:248-254

Brisson N, Giroux H, Zollinger M, Camirand A, Simard C (1989) Maturation and subcellular compartmentation of potato starch phosphorylase. Plant Cell 1:559-566

Camirand A, St-Pierre B, Marineau C, Brisson N (1990) Occurrence of a copia-like transposable element in one of the introns of the potato starch phosphorylase gene. Mol Gen Gene 224:33-39

Chan KF, Hurst MO, Graves DJ (1982) Phosphorylase kinase specificity. A comparative study with cAMP-dependent protein kinase on synthetic peptides and peptide analogs of glycogen synthase and phosphorylase. J Biol Chem 257:3655-3659

Chang SC, Lin PC, Chen HM, Wu JS, Juang RH (2000) The isolation and characterization of chaperonin 60 from sweet potato roots - involvement of the chaperonins in starch biosynthesis. Bot Bull Academia Sin 41:105-111

Chen ZJ, Parent L, Maniatis T (1996) Site-specific phosphorylation of $\mathrm{I} \kappa \mathrm{B} \alpha$ by a novel ubiquitin-dependent protein kinase activity. Cell 84:853-862

Chen HM, Chang SC, Wu CC, Cuo TS, Wu JS, Juang RH (2002) Regulation of the catalytic behaviour of L-form starch phosphorylase from sweet potato roots by proteolysis. Physiol Plant 114:506-515

Chiang C-L, Lu Y-L, Juang RH, Lee P-D, Su JC (1991) Native and degraded forms of sweet potato starch phosphorylase. Agric Biol Chem 55:641-646

Duwenig E, Steup M, Kossmann J (1997) Induction of genes encoding plastidic phosphorylase from spinach (Spinacia oleracea L.) and potato (Solanum tuberosum L) by exogenously supplied carbohydrates in excised leaf discs. Planta 203:111-120

Glotzer M, Murray AW, Kirschner MW (1991) Cyclin is degraded by the ubiquitin pathway. Nature 349:132-138

Hanes CS (1940a) The breakdown and synthesis of starch by an enzyme system from pea seeds. Proc R Soc(Lond) B 128:421-450

Hanes CS (1940b) The reversible formation of starch from glucose1-phosphate catalyzed by potato phosphorylase. Proc R Soc (Lond) B 129:174-208

Hardie DG (1999) Plant protein serine/threonine kinases: classification and functions. Annu Rev Plant Physiol Plant Mol Biol 50:97-131

Huber SC, Huber JL, Liao PC, Gage DA, McMichael RW Jr, Chourey PS, Hannah LC, Koch K (1996) Phosphorylation of serine-15 of maize leaf sucrose synthase. Occurrence in vivo and possible regulatory significance. Plant Physiol 112:793-802

Juang RH, Chang YD, Sung HY, Su JC (1984) Oven-drying method for polyacrylamide gel slab packed in cellophane sandwich. Anal Biochem 141:348-350

Kruger NJ, Ap Rees T (1983) Properties of $\alpha$-glucan phosphorylase from pea-chloroplasts. Phytochemistry 22:1891-1898
Laemmli UK (1970) Cleavage of structural proteins during the assembly of the head bacteriophage T4. Nature 227:680-685

Lin CT, Yeh KW, Lee PD, Su JC (1991) Primary structure of sweet potato starch phosphorylase deduced from its cDNA sequence. Plant Physiol 95:1250-1253

Liu TTY, Shannon JC (1981) A nonaqueous procedure for isolating starch granules with associated metabolites from maize endosperm. Plant Physiol 67:518-524

Mingo-Castel AM, Young RE, Smith OE (1976) Kinetin-induced tuberization of potato in vitro: on the mode of action of kinetin. Plant Cell Physiol 17:557-570

Mori H, Tanizawa K, Fukui T (1991) Potato tuber type H phosphorylase isoenzyme. Molecular cloning, nucleotide sequence, and expression of a full-length cDNA in Escherichia coli. J Biol Chem 266:18446-18453

Mori H, Tanizawa K, Fukui T (1993) A chimeric $\alpha$-glucan phosphorylase of plant type $\mathrm{L}$ and $\mathrm{H}$ isozymes: functional role of 78 residue insertion in type $\mathrm{L}$ isozyme. $\mathrm{J}$ Biol Chem 268:5574-5581

Nakano K, Fukui T (1986) The complete amino acid sequence of potato $\alpha$-glucan phosphorylase. J Biol Chem 261:8230-8236

Nakano K, Mori H, Fukui T (1989) Molecular cloning of cDNA encoding potato amyloplast $\alpha$-glucan phosphorylase and the structure of its transit peptide. J Biochem (Tokyo) 106:691-695

Nelson O, Pan D (1995) Starch synthesis in maize endosperms. Annu Rev Plant Physiol Plant Mol Biol 46:475-496

Rogers S, Wells R, Rechsteiner M (1986) Amino acid sequences common to rapidly degraded proteins: the PEST hypothesis Science 234:364-368

Schupp N, Ziegler P (2004) The relation of starch phosphorylases to starch metabolism in wheat. Plant Cell Physiol 45:1471-1484

Sprang SR, Acharya KR, Goldsmith EJ, Stuart DI, Varvill K, Fletterick RJ, Madsen NB, Johnson LN (1988) Structural changes in glycogen phosphorylase induced by phosphorylation. Nature 336:215-221

St-Pierre B, Brisson N (1995) Induction of the plastidic starchphosphorylase gene in potato storage sink tissue. Planta 195:339-344

Tetlow IJ, Wait R, Lu Z, Akkasaeng R, Bowsher CG, Esposito S, Kosar-Hashemi B, Morell MK, Emes MJ (2004) Protein phosphorylation in amyloplasts regulates starch branching enzyme activity and protein-protein interactions. Plant Cell 16:694-708

Van Berkel J, Conrads Strauch J, Steup M (1991) Glucan-phosphorylase forms in cotyledons of Pisum sativm L.: localization, developmental change, in vitro translation, and processing. Planta 185:432-439

Zeeman SC, Thorneycroft D, Schupp N, Chapple A, Weck M, Dunstan H, Haldimann P, Bechtold N, Smith AM, Smith SM (2004) Plastidial $\alpha$-glucan phosphorylase is not required for starch degradation in Arabidopsis leaves but has a role in the tolerance of abiotic stress. Plant Physiol 135:849-858 